\title{
The Role of Hippocampal Glutamate Receptor-A-Dependent Synaptic Plasticity in Conditional Learning: The Importance of Spatiotemporal Discontiguity
}

\author{
Wolfram B. Schmitt, ${ }^{1}$ Roozbeh Arianpour, ${ }^{1}$ Robert M. J. Deacon, ${ }^{1}$ Peter H. Seeburg, ${ }^{2}$ Rolf Sprengel, ${ }^{2}$ \\ J. Nicholas P. Rawlins, ${ }^{1}$ and David M. Bannerman ${ }^{1}$ \\ ${ }^{1}$ Department of Experimental Psychology, University of Oxford, Oxford OX1 3UD, United Kingdom, and 2Max-Planck Institute of Medical Research, \\ Department of Molecular Neurobiology, D-69120 Heidelberg, Germany
}

\begin{abstract}
Gene-targeted mice lacking the AMPA receptor subunit glutamate receptor-A (GluR-A or GluR1) and mice with cytotoxic hippocampal lesions were compared with wild-type and sham-operated controls, respectively, on a conditional learning task using an elevated T-maze. Floor inserts (white perspex vs wire mesh) provided a conditional cue indicating in which goal arm a food reward was to be found. The relationship between the floor insert and the rewarded goal arm was constant throughout the experiment. Both lesioned and knock-out mice were able to acquire the task if the floor inserts extended throughout the entire maze, including the start arm and both goal arms. In contrast, both lesioned and knock-out mice were unable to acquire the task if the floor inserts were only present in the start arm of the maze. The absence of the conditional cue (the floor insert) at the time when the place-reward association was experienced thus critically determined whether or not the mice were impaired. We suggest that hippocampal GluR-A-dependent synaptic plasticity contributes to a memory system in rodents for encoding both the spatial and temporal contexts (the where and the when) associated with a particular event.
\end{abstract}

Key words: AMPA receptors; LTP; hippocampus; conditional learning; T-maze; mice

\section{Introduction}

The amnestic effects of hippocampal lesions are well documented, leading to the widely held belief that the hippocampus is crucial for learning and memory (Scoville and Milner, 1957; Vargha-Khadem et al., 1997). The rodent hippocampus has been especially implicated in spatial learning (O'Keefe and Nadel, 1978; Morris et al., 1982). However, it is also important for encoding the temporal sequence of events (Agster et al., 2002; Fortin et al., 2002; Kesner et al., 2002). The rodent hippocampal memory system may thus provide the means for encoding both the spatial and temporal contexts (the where and the when) associated with a particular event (Morris et al., 2003; Eichenbaum and Fortin, 2003).

It is widely believed that memories are stored as changes in the strength of synaptic connections between neurons (Hebb, 1949). Long-term potentiation (LTP) is an experimental model of a type of synaptic plasticity that may underlie memory storage (Bliss and Lomo, 1973; Bliss and Collingridge, 1993; Martin et al., 2000). Induction of the most commonly studied form of hip-

Received March 24, 2004; revised May 26, 2004; accepted June 20, 2004.

This work was supported by a European Union Framework V grant (QLG 3-CT-1999-01022). This paper is dedicated to the memory of Roozbeh Arianpour (1980 - 2003). We thank Greg Daubney and Dr. Jonathan Flint for their assistance with genotyping.

Correspondence should be addressed to D. M. Bannerman, Department of Experimental Psychology, University of Oxford, South Parks Road, 0xford 0X1 3UD, UK. E-mail: david.bannerman@psy.ox.ac.uk.

DOI:10.1523/JNEUROSCI.1093-04.2004

Copyright $\odot 2004$ Society for Neuroscience $\quad$ 0270-6474/04/247277-06\$15.00/0 pocampal LTP requires the activation of NMDA receptors (Collingridge et al., 1983). The resulting long-term expression, however, appears to rely on recruiting additional AMPA receptors containing glutamate receptor-A (GluR-A; GluR1) subunits to the activated synapse (Malinow and Malenka, 2002). Mice lacking the GluR-A subunit no longer develop conventional CA3CA1 LTP (Zamanillo et al., 1999). Somewhat surprisingly, these mice displayed normal, hippocampus-dependent spatial reference memory (Zamanillo et al., 1999; Reisel et al., 2002; Schmitt et al., 2003, 2004). They did, however, resemble hippocampallesioned animals in displaying a profound spatial working memory impairment (Reisel et al., 2002; Schmitt et al., 2003).

In working memory tasks such as nonmatching to place (NMTP) on the T-maze (Rawlins and Olton, 1982), the animal's response must change from trial to trial, depending on trialspecific sample information given immediately beforehand. The visit to the sample goal arm provides the trial-specific conditional cue that determines which arm should subsequently be chosen during the choice trial. The present study further examined the role of hippocampal GluR-A-dependent synaptic plasticity in conditional learning on the T-maze. The conditional cue was now provided by different, interchangeable floor inserts (white perspex vs wire mesh). By varying the extent of the floor inserts, it is possible to compare performance under conditions in which the conditional cue is either present (inserts extend throughout the entire maze) or absent (inserts limited to the start arm only) when the animal experiences a particular place-reward associa- 
tion. It is thus possible to manipulate the degree of spatiotemporal contiguity between the conditional cue and the choice outcome, to require or eliminate the need to retrieve the appropriate conditional cue memories needed to learn the task contingencies (Rawlins, 1985). GluR-A ${ }^{-1-}$ and hippocampal-lesioned mice were therefore compared with wild-type and sham-lesioned controls on two versions of the conditional T-maze task in which the floor inserts were either limited to the start arm only or extended throughout the entire maze.

\section{Materials and Methods}

Subjects. Experiments 1 and 3 each consisted of two groups of C57BL/ 6Jola Hsd female mice obtained from Harlan OLAC Ltd., (Oxon, UK), which had received either bilateral NMDA-induced cytotoxic hippocampal lesions or sham surgery [see Deacon et al. (2002) for details of methodology]. Experiments 2 and 4 were conducted with separate cohorts of experimentally naive, age-matched, female, wild-type, and GluRA ${ }^{-1-}$ mice that were bred in the Department of Experimental Psychology at the University of Oxford [see Zamanillo et al. (1999) for details of genetic construction, breeding, and subsequent genotyping]. The genetic background of these mice consisted of a cross between C57BL/6JolaHsd and 129S2/SvHsd strains. Mice were housed in group cages and tested during the light phase of the day (from 7:00 A.M. to 7:00 P.M.). Mice had ad libitum access to water but were maintained on a restricted feeding schedule at $85 \%$ of their free-feeding weight throughout behavioral testing. All experiments were conducted under the auspices of UK Home Office Project and Personal licenses held by the authors.

Apparatus. Learning was assessed on an elevated wooden T-maze, consisting of a start arm $(47 \times 10 \mathrm{~cm})$ and two identical goal arms $(35 \times 10$ $\mathrm{cm}$ ), surrounded by a $10-\mathrm{cm}$-high wall. A metal food well was located 3 $\mathrm{cm}$ from the end of each goal arm. The maze was located $1 \mathrm{~m}$ above the floor in a well lit laboratory that contained various prominent distal extramaze cues. Floor inserts of either white perspex or wire mesh $(5 \times 5$ $\mathrm{mm}$ mesh, affixed to a gray painted, wooden backing) could be placed into the maze according to the counterbalanced experimental protocol.

Experiments 1 and 2. Experiment 1 comprised hippocampal (HPC)lesioned $(n=6)$ and sham-lesioned mice $(n=6)$. The mice were first habituated to drinking sweetened, condensed milk (diluted 50:50 with water) on two arms of an elevated Y-maze (Reisel et al., 2002) in their colony holding room (i.e., not the testing room). Once all the mice were running freely on the Y-maze and readily consuming the milk rewards, testing on the T-maze began.

Mice were trained on a conditional learning task in which a floor insert $(57 \times 10 \mathrm{~cm})$, covering the whole of the start arm and extending right across to the wall opposite the start arm at the junction of the maze, acted as a conditional cue or occasion setter. For half of the sham- and HPClesioned animals, the presence of the white perspex insert indicated that the $0.1 \mathrm{ml}$ milk reward was available in the left goal arm. In contrast, the reward was in the right goal arm if the start arm contained the wire mesh, floor insert. For the remaining sham- and HPC-lesioned mice, the opposite pair of floor insert-reward contingencies applied (e.g., perspexright, wire-left). The relationship between the floor insert and the rewarded goal arm was constant for each animal throughout the experiment. Mice received 16 test sessions comprising 10 trials per session with an intertrial interval of 5-10 min. Each session consisted of five trials with each of the two floor inserts, and no more than three consecutive trials with the same floor insert, according to a pseudorandom sequence. During the final session of 10 trials the milk reward was delivered into the food well only after the mouse had made a choice. This was to ensure that the mice were unable to solve the task by smelling the milk reward.

On completion of the start arm conditional learning task, spatial working memory was assessed in both the sham- and hippocampallesioned mice. They received 20 trials of discrete trial, rewarded alternation (NMTP) testing using the same elevated T-maze but with no floor inserts present. Each trial consisted of a sample run and a choice run. On the sample run the mice were forced either left or right by a wooden block to obtain a milk reward, according to a pseudorandom sequence (with equal numbers of left and right turns per session, and with no more than three consecutive turns in the same direction). The block was then removed and the mouse was placed, facing the experimenter, at the end of the start arm and allowed a free choice of either arm. The time interval between the sample run and the choice run was $\sim 15 \mathrm{sec}$. The mouse was rewarded for choosing the previously unvisited arm (i.e., for alternating).

Experiment 2 consisted of wild-type $(n=7)$ and GluRA ${ }^{-1-}$ mice $(n=$ 7). Habituation and testing were the same as for experiment 1 . Mice received a total of 14 test sessions. On completion of the start arm conditional task, the wild-type (WT) and GluRA ${ }^{-1-}$ mice were trained on a simple discrimination using goal arm, floor inserts made of either white perspex or wire mesh, using the same elevated T-maze. The mice were trained to discriminate between the white perspex floor insert and the wire mesh insert for a milk reward. The mice received eight sessions, each consisting of 10 trials. One goal arm, floor insert was designated as always rewarded throughout testing, and was counterbalanced across groups. The right-left orientation of the floor inserts was determined according to a pseudorandom sequence, with the reward in the left goal arm on five trials of each session, and with no more than three consecutive trials rewarded in the left or right goal arm.

Experiments 3 and 4. Experiment 3 comprised HPC- $(n=6)$ and sham-lesioned mice $(n=6)$. After habituation (see above), the mice were tested as in experiments 1 and 2 with the only exception that the floor inserts now extended throughout the entire maze, including the start arm and both of the goal arms. As in experiment 1, half of the mice were trained so that when the white perspex insert was in place, the reward was in the left goal arm, and when the wire mesh insert was present, the reward was in the right goal arm. For the remaining animals the opposite pair of floor insert-reward contingencies applied (e.g., perspex-right, wire-left). Mice received 13 test sessions. Otherwise the testing procedure was identical to Experiments 1 and 2. Experiment 4 consisted of wild-type $(n=8)$ and GluRA ${ }^{-1-}$ mice $(n=8)$. Habituation and testing were the same as for experiment 3 . Mice received 11 test sessions in total.

On completion of the full maze, floor insert task, spatial working memory was assessed in both of these cohorts of mice (sham-hippocampal and wild-type-knock-out). They received 20 trials of discrete trial, rewarded alternation testing using the same elevated T-maze but with no floor inserts present.

Histology. On completion of behavioral testing mice with sham or HPC lesions were anesthetized and perfused transcardially with physiological saline followed by $10 \%$ formol-saline. The brains were removed and stored in formol-saline. They were then placed in a $30 \%$ sucroseformalin solution for $24 \mathrm{hr}$, frozen, sectioned, and stained with cresyl violet.
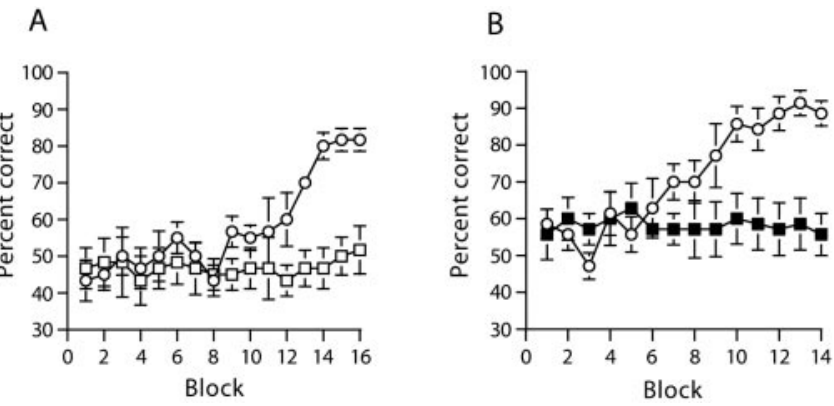

Figure 1. Mice with hippocampal lesions and GluR-A ${ }^{-/-}$mice are impaired on a conditional version of the T-maze task in which floor inserts located in the start arm of the maze act as conditional cues, indicating which goal arm is to be rewarded. Mean percentage of correct responses \pm SEM (untransformed, 10 trials per block) for mice with cytotoxic hippocampal lesions (white squares) or sham-operated controls (white circles) $(A)$ and GluR-A ${ }^{-1-}$ mice (black squares) and wild-type controls (white circles) $(B)$. During the last block of 10 trials of each experiment, the reward was delivered only after the animal had made a choice. Where error bars appear to be absent, the error is too small to be visible. 


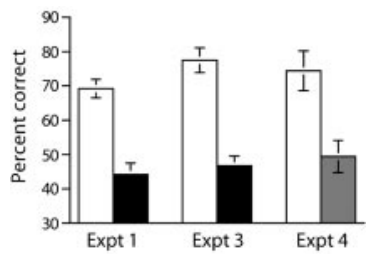

Figure 2. Mice with hippocampal lesions and GluR-A ${ }^{-1-}$ mice are impaired on a discrete trial, rewarded alternation (spatial nonmatching to place) task on the elevated T-maze (no floor inserts present). Mean percentage of correct responses \pm SEM of 20 trials for experiment 1 (left) and experiment 3 (center), sham-operated controls (white bar) and mice with cytotoxic hippocampal lesions (black bar), and experiment 4 (right), wild-type controls (white bar) and GluR-A ${ }^{-1-}$ mice (gray bar).

\section{Results}

Experiments 1 and 2

Sham-operated mice learned to use the start arm floor insert as a conditional cue to solve the task (Fig. $1 A$, experiment 1 ). In contrast, mice with hippocampal lesions still displayed chance levels of performance even after 160 trials. Because the data were not normally distributed, they were subjected to a "square" transformation. An ANOVA of the transformed data revealed a main effect of group $\left(F_{(1,10)}=26.0 ; p<0.001\right)$, a main effect of session $\left(F_{(15,150)}=5.1 ; p<0.001\right)$, and a groups by sessions interaction $\left(F_{(15,150)}=4.1 ; p<0.001\right)$. Subsequent analysis of simple main effects showed that the hippocampal-lesioned mice were significantly impaired relative to the sham controls from sessions 12-16 (all $\left.F_{(1,157)}>5.6 ; p<0.05\right)$. The hippocampal-lesioned mice were also impaired during NMTP testing using the same elevated T-maze but in the absence of any floor inserts ( $2.7 \%$; $\mathrm{HPC}=44.2 \pm 3.3 \% ; t_{(10)}=5.9 ; p<0.0005$ ) (Fig. 2, experiment 1).

WT mice, like sham-operated controls, were able to solve the start arm, floor insert version of the task (Fig. $1 B$, experiment 2). GluRA $^{-1-}$ mice, like mice with HPC lesions, were unable to do so. The knock-out animals were still at chance even after 140 trials. An ANOVA revealed a significant main effect of session $\left(F_{(13,156)}=5.3 ; p<0.0001\right)$, a significant main effect of group $\left(F_{(1,12)}=5.3 ; p<0.05\right)$, and a significant groups by sessions interaction $\left(F_{(13,156)}=5.9 ; p<0.0001\right)$. Subsequent analysis of simple main effects confirmed that this was attributable to the fact that the GluRA ${ }^{-/-}$mice were significantly impaired relative to the WT mice from sessions $9-14\left(\right.$ all $\left.F_{(1,50)}>5.7 ; p<0.05\right)$. The same GluRA ${ }^{-1-}$ mice were, however, able to solve a simple discrimination task based on floor inserts limited just to the goal arms (Fig. 3). Both WT and knock-out mice acquired the simple discrimination task at a similar rate. There was a significant main effect of session $\left(F_{(7,84)}=21.2 ; p<0.001\right)$, but neither a main effect of group $(F<1 ; p>0.20)$, nor a groups by sessions interaction $\left(F_{(7,84)}=1.02 ; p>0.20\right)$.

It is worth noting that the general level of performance was higher in experiment 2 compared with experiment 1 . This may have been attributable to differences in the background strains used in the two studies (see Subjects), but could equally be caused by other uncontrolled differences between the cohorts. This was reflected in a significant overall main effect of experiment $\left(F_{(1,22)}=18.74 ; p<0.0005\right)$. Importantly, however, there was no interaction between experiment and experimental manipulation (sham-operated control or wild-type control vs either HPC lesion or GluRA ${ }^{-/-}$mice), or an experiment by experimental manipulation by blocks interaction (both $F<1$ ). Hence, there was no difference in the effects of the manipulations in the two experiments.

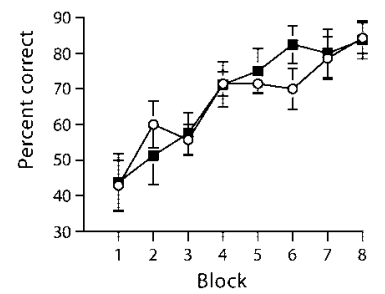

Figure 3. Wild-type and GluR-A ${ }^{-1-}$ mice acquire a simple discrimination using floor inserts located in the goal arms of the maze at the same rate. Mean percentage of correct responses \pm SEM (10 trials per block) for GluR-A ${ }^{-1-}$ mice (black squares) and wild-type controls (white circles).

\section{A}

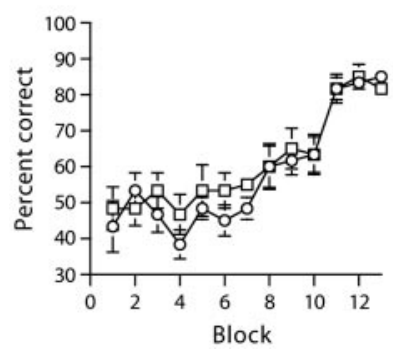

Figure 4. Mice with hippocampal lesions and GluR-A ${ }^{-1-}$ mice are able to acquire a conditional version of the T-maze task in which floor inserts extending throughout the entire maze (the start arm and both goal arms) act as conditional cues, indicating which goal arm is to be rewarded. Mean percentage of correct responses \pm SEM (10 trials per block) for mice with cytotoxic hippocampal lesions (white squares) or sham-operated controls (white circles) $(A)$ and GluR-A ${ }^{-1-}$ mice (black squares) and wild-type controls (white circles) (B). During the last block of 10 trials of each experiment the reward was delivered only after the animal had made a choice. Where error bars appear to be absent, the error is too small to be visible.

\section{Experiments 3 and 4}

Both sham- and HPC-lesioned animals were able to solve the conditional task when the floor inserts extended throughout the entire maze (Fig. $4 \mathrm{~A}$, experiment 3 ). Both groups of mice learned the task at a similar rate. These impressions were confirmed by an ANOVA that showed a significant main effect of session $\left(F_{(12,120)}=19.3 ; p<0.0001\right)$, but neither a main effect of group $\left(F_{(1,10)}=2.6 ; p>0.10\right)$, nor a groups by sessions interaction $(F<$ $1 ; p>0.20)$.

Likewise, both WT and GluRA ${ }^{-1-}$ mice were also able to successfully solve the full maze insert version of the task (Fig. $4 B$, experiment 4). Again, an ANOVA revealed a significant main effect of session $\left(F_{(10,140)}=13.0 ; p<0.0001\right)$, but neither a main effect of group $\left(F_{(1,14)}=2.2 ; p>0.10\right)$, nor a groups by sessions interaction $(F<1 ; p>0.20)$.

Both of these HPC lesion and GluRA ${ }^{-1-}$ groups of mice did, however, display a spatial working memory impairment when subsequently tested on a discrete trial, rewarded alternation (NMTP) task using the same elevated T-maze but without any floor inserts. Mice with HPC lesions were impaired relative to sham-operated controls ( ham $=77.5 \pm 3.6$; $\mathrm{HPC}=46.7 \pm 2.8$; $t_{(10)}=6.8 ; p<0.0001$ ) (Fig. 2, experiment 3). Similarly, whereas wild-type mice showed good levels of rewarded alternation $(74.4 \pm 5.8 \%)$, the GluRA ${ }^{-1-}$ mice remained at chance $(49.4 \pm$ 4.7\%; $U=55.0 ; p<0.05$ ) (Fig. 2, experiment 4).

\section{Histology}

All mice in the HPC lesion groups of experiments 1 and 3 possessed near complete hippocampal lesions that encompassed 

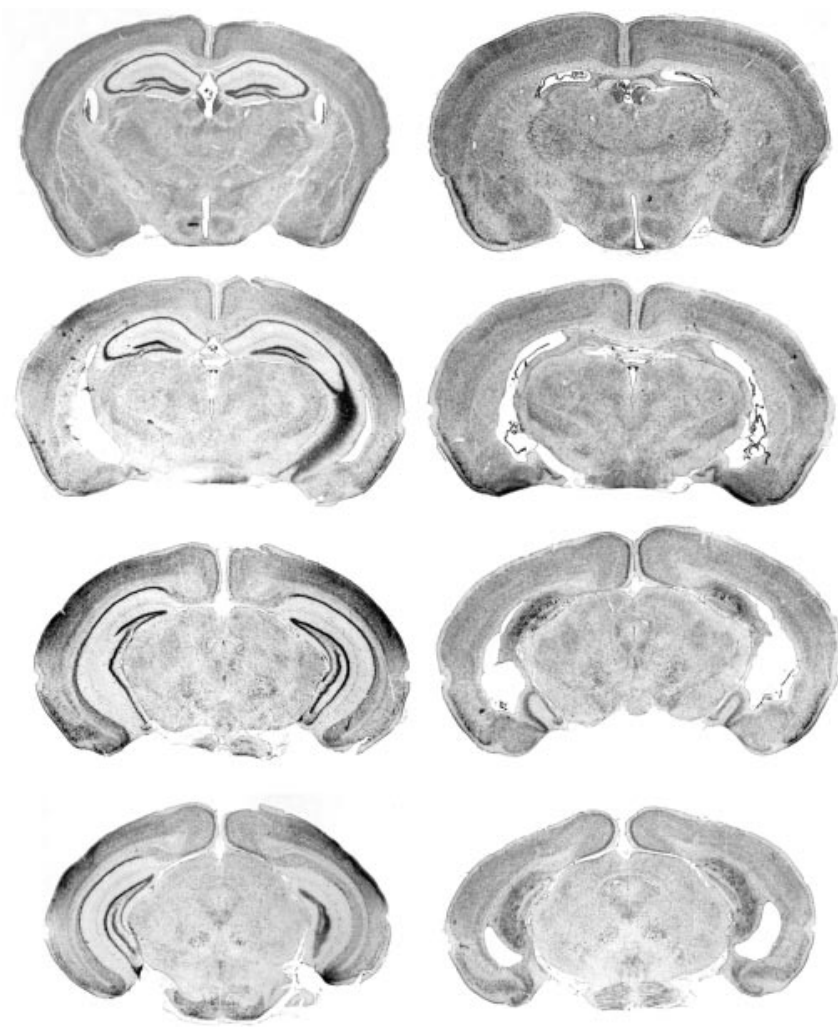

Figure 5. Photomicrographs of coronal sections taken from a sham-operated control animal (left) and a representative (median for size) hippocampal-lesioned mouse (right).

both the dorsal and ventral hippocampus (Fig. 5). There was only minimal damage to the subicular complex and no observable damage to the entorhinal cortex or to the thalamus. There was no difference in the size of the lesions in mice who participated separately in experiments 1 and 3 .

\section{Discussion}

Mice with hippocampal lesions were unable to acquire a conditional T-maze task in which floor inserts present in the start arm of the maze provided trial-specific information indicating which goal arm should be chosen to obtain reward (Murray and Ridley, 1999). The performance of the lesioned animals remained at chance even after 160 trials. In contrast, if the floor inserts extended throughout the entire maze, including the start arm and both goal arms, then mice with hippocampal lesions were able to acquire the task successfully. A strikingly similar pattern of results was obtained with GluR-A ${ }^{-1-}$ mice. The knock-out animals were able to acquire the task when the floor inserts were present throughout the maze, but were unable to do so when the conditional cue was limited to the start arm only. The performance of the knock-out mice remained at chance on this version of the task, even after 140 trials. These results show that GluR-A ${ }^{-1-}$ mice, like mice with hippocampal lesions, are impaired on a conditional version of the T-maze task, but only if the conditional cue and the place reward association are spatiotemporally discontiguous. The absence of the conditional cue (the floor insert) at the time when the place-reward association was experienced critically determined whether or not lesioned and knock-out mice were impaired. Although no response latencies were recorded, the time taken for the mouse to get from the start arm to the reward was typically $1-4 \mathrm{sec}$. When the maze inserts extended throughout the entire maze, so that the conditional cue was present when the mouse experienced the place-reward association, then both groups of mice acquired the task as well as their respective control groups.

These results substantially extend our previous findings with GluR-A ${ }^{-1-}$ mice. There are several important differences between the NMTP version of the T-maze task (Reisel et al., 2002), and the "start arm" conditional task described in the present study that are worth noting. First, the conditional cue in the present study is nonspatial in nature (the floor inserts), whereas during NMTP testing the conditional information is provided by the spatial location of the goal arm visited on the sample run. Second, in contrast to NMTP testing, in the present study the conditional cue is present at the choice point when the animal makes a response. Third, the conditional learning task is acquired gradually in an incremental manner by the controls, in contrast to the very rapid, often one-trial acquisition associated with working memory performance on tasks such as NMTP. In this respect, the acquisition of the start arm conditional task more resembles the incremental acquisition of spatial reference memory tasks such as the water maze, although crucially these two tasks are very different in terms of their requirement for GluR-Adependent synaptic plasticity. Importantly, the "start arm only" and "full maze insert" versions of the conditional task in the present study are very closely matched for control difficulty, unlike standard spatial working and reference memory versions of the three arm maze task (Reisel et al., 2002). Fourth, the present results demonstrate the importance of the spatiotemporal discontiguity between the conditional cue and the place-reward association in terms of whether or not GluR-A-dependent synaptic plasticity is required. Any theory of GluR-A function must explain these new findings, in addition to the striking spatial working memory impairment displayed by GluR-A ${ }^{-1-}$ mice.

One possible account is that GluR-A-dependent synaptic plasticity in the hippocampus underlies a temporal tagging mechanism that provides the ability to encode what happened when. On the start arm conditional task, when the mouse makes the correct choice of goal arm and finds a reward, it must link that outcome to the immediately preceding experience of a particular start arm insert that must therefore be temporally tagged to differentiate the present trial from the previous one. This is not necessary if the conditional cue is present at the time when the animal experiences the place-reward association, as is the case when the inserts cover the whole maze. Indeed, under these conditions it is possible that the mice do not in fact solve the task using a conditional operation, but rather they do so in a nonconditional manner, deconstructing the task into discrete go-no-go discriminations (Deacon et al., 2001).

A role for GluR-A-dependent synaptic plasticity in the temporal tagging of events could also explain the striking spatial working memory impairment in the GluR-A ${ }^{-1-}$ mice (Reisel et al., 2002; Schmitt et al., 2003). For example, along similar lines to the start arm conditional task, during NMTP testing on the T-maze the mouse must temporally tag its visit to the goal arm during the sample run to differentiate the present trial from previous ones. Likewise, during radial maze performance, to avoid making working memory errors an animal must be able to keep track of which arms have been visited on that particular trial. It is therefore essential to be able to encode when a particular arm was visited to be able to separate the present trial from previous visits to the maze.

The idea that the hippocampus is important for the temporal encoding of events (what happened when) is not new. A number of recent studies have shown that rats with hippocampal lesions 
are unable to encode the temporal order in which they are exposed to a series of odors (Fortin et al., 2002; Kesner et al., 2002). We now suggest that this temporal encoding of events is mediated by hippocampal, GluR-A-dependent synaptic plasticity. There is evidence that deficits in NMDA receptor-dependent LTP are associated with impairments on tasks in which the temporal encoding or tagging of events is likely to be of crucial importance. In addition to deficits on spatial working memory tasks (Tonkiss and Rawlins, 1991; Steele and Morris, 1999; Lee and Kesner, 2002), rats or mice lacking NMDA-receptor-dependent LTP are also impaired on nonspatial tasks in which the temporal tagging or encoding of events is likely to be crucial for normal performance (Tonkiss et al., 1988; Huerta et al., 2000; Rondi-Reig et al., 2001).

An important question that remains to be answered is whether the role of hippocampal GluR-A-dependent synaptic plasticity extends beyond the ability to discriminate between events on the basis of when they occurred. One version of the temporal tagging hypothesis is that the role of GluR-A-dependent synaptic plasticity is strictly limited to the temporal encoding of events and is not required for learning to associate a particular spatial location with a particular outcome or response-outcome contingency. Thus, GluR-A ${ }^{-1-}$ mice can generate spatial representations of the arms they have visited during radial maze or T-maze testing, but cannot encode when those arms were visited. This spatial or contextual retrieval of events (encoding what happened where, regardless of when; Hirsh, 1974; Good and Honey, 1991) may be supported by residual, GluR-A-independent forms of LTP in the hippocampus (Hoffman et al., 2002), and is sufficient for GluR$\mathrm{A}^{-1-}$ mice to be able to acquire spatial reference memory tasks such as the water maze (Zamanillo et al., 1999; Reisel et al., 2002; Schmitt et al., 2003, 2004). An alternative version of the theory, which would also be consistent with the data set, is that the role of rapid-onset, GluR-A-dependent synaptic plasticity extends beyond just the temporal tagging of events and in fact underlies a one-trial, ability to encode both where and when events or episodes happened. This version of the theory must then also include a separate GluR-A-independent hippocampal mechanism for the incrementally acquired spatial or contextual retrieval of information, as typified by spatial reference memory acquisition.

To conclude, GluR-A-dependent synaptic plasticity in the hippocampus plays an essential role during acquisition of a conditional version of the T-maze task, but only if the conditional cue and the place-reward association are spatiotemporally discontiguous. The absence of the conditional cue (the floor insert) at the time when the place-reward association was experienced critically determined whether or not lesioned and GluR-A knockout mice were impaired. These results could potentially be explained by a role for GluR-A-dependent, hippocampal synaptic plasticity in the temporal tagging or encoding of events (what happened when), an account which would also explain the striking spatial working memory impairment displayed by these animals. Equally the results could also potentially be explained if GluR-A-dependent synaptic plasticity underlies a one-trial, ability to encode what happened where. If the role of GluR-Adependent synaptic plasticity is strictly limited to temporal tagging then one-trial, spatial working memory should improve as the need for temporal tagging is reduced. If GluR-A underlies the ability to encode what happened where, then spatial working memory will be impaired regardless of whether or not there is potential interference from previous trials. Further experiments are required to distinguish between these possibilities and to establish the precise role of NMDA receptors in these distinct as- pects of hippocampal information processing that we have now repeatedly differentiated (Morris et al., 1986; Bannerman et al., 1995; Saucier and Cain, 1995; Tsien et al., 1996; Good and Bannerman, 1997).

\section{References}

Agster KL, Fortin NJ, Eichenbaum H (2002) The hippocampus and disambiguation of overlapping sequences. J Neurosci 22:5760-5768.

Bannerman DM, Good MA, Butcher SP, Ramsay M, Morris RGM (1995) Distinct components of spatial learning revealed by prior training and NMDA receptor blockade. Nature 378:182-186.

Bliss TVP, Collingridge GL (1993) A synaptic model of memory: long-term potentiation in the hippocampus. Nature 361:31-39.

Bliss TVP, Lømo T (1973) Long-lasting potentiation of synaptic transmission in the dentate area of anaesthetized rabbit following stimulation of the perforant path. J Physiol (Lond) 232:331-356.

Collingridge GL, Kehl SJ, McLennan H (1983) Excitatory amino acids in synaptic transmission in the Schaffer collateral-commissural pathway of the rat hippocampus. J Physiol (Lond) 334:33-46.

Deacon RMJ, Bannerman DM, Rawlins JNP (2001) Conditional discriminations based on external and internal cues in rats with cytotoxic hippocampal lesions. Behav Neurosci 115:43-57.

Deacon RMJ, Bannerman DM, Kirby BP, Croucher A, Rawlins JNP (2002) The effects of cytotoxic hippocampal lesions in mice on a cognitive test battery. Behav Brain Res 133:57-68.

Eichenbaum H, Fortin N (2003) Episodic memory and the hippocampus: it's about time. Curr Dir Psychol Sci 12:53-57.

Fortin NJ, Agster KL, Eichenbaum HB (2002) Critical role of the hippocampus in memory for sequences of events. Nat Neurosci 5:458-462.

Good MA, Bannerman DM (1997) Differential effects of ibotenic acid lesions of the hippocampus and blockade of $N$-methyl-D-aspartate receptor-dependent long-term potentiation on contextual processing in rats. Behav Neurosci 111:1171-1183.

Good M, Honey RC (1991) Conditioning and contextual retrieval in hippocampal rats. Behav Neurosci 105:499-509.

Hebb DO (1949) The organisation of behaviour. New York: John Wiley.

Hirsh R (1974) The hippocampus and contextual retrieval of information from memory: a theory. Behav Biol 12:421-444.

Hoffman D, Sprengel R, Sakmann B (2002) Molecular dissection of associative plasticity in CA1 hippocampal pyramidal neurons. Proc Natl Acad Sci USA 99:7740-7745.

Huerta PT, Sun LD, Wilson MA, Tonegawa S (2000) Formation of temporal memory requires NMDA receptors within CA1 pyramidal neurons. Neuron 25:473-480.

Kesner RP, Gilbert PE, Barua LA (2002) The role of the hippocampus in memory for the temporal order of a sequence of odors. Behav Neurosci 116:286-290.

Lee I, Kesner RP (2002) Differential contribution of NMDA receptors in hippocampal subregions to spatial working memory. Nat Neurosci 5:162-168.

Malinow R, Malenka RC (2002) AMPA receptor trafficking and synaptic plasticity. Annu Rev Neurosci 25:103-126.

Martin SJ, Grimwood PD, Morris RGM (2000) Synaptic plasticity and memory: an evaluation of the hypothesis. Annu Rev Neurosci 23:649-711.

Morris RGM, Garrud P, Rawlins JNP, O’Keefe J (1982) Place navigation impaired in rats with hippocampal lesions. Nature 297:681-683.

Morris RGM, Anderson E, Lynch GS, Baudry M (1986) Selective impairment of learning and blockade of long-term potentiation by an $\mathrm{N}$-methylD-aspartate receptor antagonist, AP5. Nature 319:774-776.

Morris RGM, Moser EI, Riedel G, Martin SJ, Sandin J, Day M, O’Carroll C (2003) Elements of a neurobiological theory of the hippocampus: the role of activity-dependent synaptic plasticity in memory. Philos Trans R Soc Lond B Biol Sci 358:773-786.

Murray TK, Ridley RM (1999) The effect of excitotoxic hippocampal lesions on simple and conditional discrimination learning in the rat. Behav Brain Res 99:103-113.

O'Keefe J, Nadel L (1978) The hippocampus as a cognitive map. Oxford: Oxford UP.

Rawlins JNP (1985) Associations across time: the hippocampus as a temporary memory store. Brain Behav Sci 8:479-496.

Rawlins JNP, Olton DS (1982) The septo-hippocampal system and cognitive mapping. Behav Brain Res 5:331-358. 
Reisel D, Bannerman DM, Schmitt WB, Deacon RMJ, Flint J, Borchardt T, Seeburg PH, JNP Rawlins (2002) The spatial memory deficit in GluR1 deficient mice. Nat Neurosci 5:868-873.

Rondi-Reig L, Libbey M, Eichenbaum H, Tonegawa S (2001) CA1-specific $\mathrm{N}$-methyl-D-aspartate receptor knockout mice are deficient in solving nonspatial transverse patterning task. Proc Natl Acad Sci USA 98:3543-3548.

Saucier D, Cain DP (1995) Spatial learning without NMDA receptordependent long-term potentiation. Nature 378:186-189.

Schmitt WB, Deacon RMJ, Seeburg PH, Rawlins JNP, Bannerman DM (2003) A within subjects, within task demonstration of intact spatial reference memory and impaired spatial working memory in GluRA deficient mice. J Neurosci 23:3953-3959.

Schmitt WB, Deacon RMJ, Reisel D, Seeburg PH, Rawlins JNP, Bannerman DM (2004) Spatial reference memory in GluR-A deficient mice using a novel, hippocampal-dependent paddling pool escape task. Hippocampus 14: 216-223.

Scoville WB, Milner B (1957) Loss of recent memory after bilateral hippocampal lesions. J Neurol Neurosurg Psychiat 20:11-21.

Steele RJ, Morris RGM (1999) Delay-dependent impairment of a matching- to-place task with chronic and intrahippocampal infusion of the NMDA antagonist D-AP5. Hippocampus 9:118-136.

Tonkiss J, Morris RGM, Rawlins JNP (1988) Intra-ventricular infusion of the NMDA antagonist AP5 impairs performance on a non-spatial operant DRL task in the rat. Exp Brain Res 73:181-188.

Tonkiss J, Rawlins JNP (1991) The competitive NMDA antagonist, AP5, but not the non-competitive antagonist, MK-801, induces a delay-related impairment in spatial working memory in rats. Exp Brain Res 85:349-358.

Tsien JZ, Huerta PT, Tonegawa S (1996) The essential role of hippocampal CA1 NMDA receptor-dependent synaptic plasticity in spatial memory. Cell 87:1327-1338.

Vargha-Khadem F, Gadin DG, Watkins KE, Connelly A, Van Paesschen W, Mishkin M (1997) Differential effects of early hippocampal pathology on episodic and semantic memory. Science 277:376-380.

Zamanillo D, Sprengel R, Hvalby O, Jensen V, Burnashev N, Rozov A, Kaiser KM, Koster HJ, Borchardt T, Worley P, Lubke J, Frotscher M, Kelly PH, Sommer B, Andersen P, Seeburg PH, Sakmann B (1999) Importance of AMPA receptors for hippocampal synaptic plasticity but not for spatial learning. Science 284:1805-1811. 